\title{
Research on Vehicle Classification Algorithm Based on Information Fusion of Dual Reluctance Sensor
}

\author{
Xu LEI ${ }^{1}$, Shuai WANG ${ }^{2}$, Li-wang $\mathrm{MA}^{3}$ and RongHE ${ }^{4}$ \\ ${ }^{1234}$ Chang'an University, Xi'an 710064, China
}

\begin{abstract}
Keywords: Anisotropic Magneto ResistanceSensor, Information Fusion, Maximum Likelihood Estimation, Vehicle Classification.
\end{abstract}

\begin{abstract}
In view of some errors existing in the vehicle detection system of single sensor node, a vehicle detection algorithm based on dual sensor information fusion for vehicle classification is proposed. The single sensor signal is detected by double threshold method, then matching the vehicle information detected from different sensor nodes within the network. Finally, to get the characteristic information of the vehicle, the maximum likelihood estimation algorithm is used to fuse the matching vehicle information. Hierarchical decision tree algorithm is used to classify the models. After the actual Lane field experimental test, the algorithm has better classification results compared with the results of a single sensor node models.
\end{abstract}

\section{Introduction}

Vehicle detector as an important infrastructure of Intelligent Transportation System(ITS), is an important source of getting traffic flow information (Vehicle flow, Lane occupancy, Vehicle speed and Vehicle type).The vehicle detector based on the magnetic resistance sensor has become a hot research field with the advantages of small size, convenient installation, maintenance, high detection precision, and strong adaptability to environment [1].

SaowaluckKaekamnerd et al. proposed the hierarchical decision tree classification algorithm, which is suitable for micro controller and has small calculation, by analyzing the characteristics of geomagnetic vehicle signal [2,3].BogenCai et al. proposed a vehicle classification algorithm based on support vector machine by extracting the feature vector [4,5]. Soner Meta et al. used BP neural network algorithm for vehicle classification, regarding feature vector obtained from acquisition waveform as the input of algorithm [6].

In some of the more congested roads, the existing classification algorithm results are not optimistic.There are two kinds of detection errors: (1)Misjudging a large car as small cars; (2)Misjudging small cars as a large car. In order to improve the detection accuracy, for the identification error of the single sensor node in the vehicle classification, this paper presents a vehicle detection method which is suitable for the vehicle classification, and uses the hierarchical decision tree algorithm to classify the vehicle.At last, the experiment can be carried out. 


\section{Vehicle Signal Acquisition}

\section{Information Acquisition}

The magnetic resistance sensor is able to convert the geomagnetic disturbance signal into electrical signal. By detecting the change of the output signal of the magnetic sensor, the micro controller can obtain the signal of the vehicle by using the magnetic sensing algorithm. In order to analysis the signal, with a series of sampled points as the three axis signals of the vehicle, with the magnetic field strength as the longitudinal axis, with time as the horizontal axis, the geomagnetic signal can be expressed by formula (1).

$$
\left\{\begin{array}{l}
X=\{X(1), X(2), X(3), \ldots, X(n)\} \\
Y=\{Y(1), Y(2), Y(3), \ldots, Y(n)\} \\
Z=\{Z(1), Z(2), Z(3), \ldots, Z(n)\}
\end{array}\right.
$$

Existing vehicle detection algorithms do not consider the problems that the large car was misjudged as small cars or small cars were misjudged as the large car, thus these result in lower classification accuracy of models. In this paper, a double threshold vehicle detection algorithm (DTVDA) based on the weighted variance weighting of vehicles is proposed. DTVDA test vehicle process is shown in Fig.1.

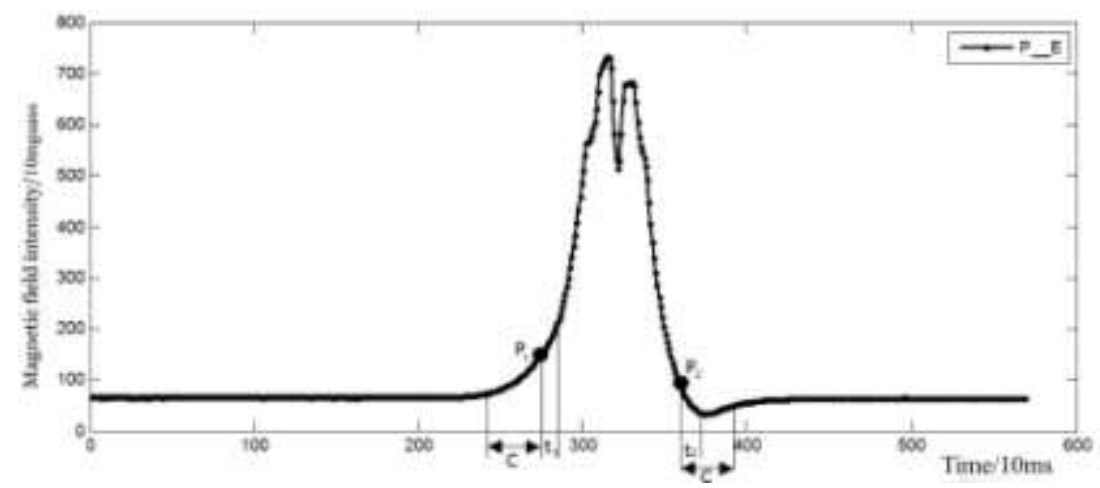

Figure1. DTVDA detection waveform of moving vehicles

\section{Signal Segmentation}

When the signal reaches the threshold value of car's arriving, it's the arrival time. When the signal reaches the threshold value of car's leaving, it's the leaving time. Because the intercept signal will cause the loss of the signal, we need to detect the arrival and departure time of the vehicle to compensate for the signal. So we address this problem by extending the measurement signal forward and backward 10 points.

\section{Vehicle Classification}

\section{Feature Parameter Extraction for Vehicle Classification}

Considering that the vehicle speed, equipment and other actual factors may have influences in same vehicle signal waveform, we selected four feature vectors, the relative length, the normalized peak (trough) number, waveform total energy and the average energy as the basis for classification models. 


\section{(1) The relative length}

Using the double sensor model mentioned above to collect time difference that the same car passes sensor nodes A and B, normalization the waveform to eliminate the effect of vehicle speed on wave length, the wave length is proportional to the length of the vehicle. Assuming $t_{\text {aarr }} t_{\text {alea }} t_{\text {barr }} t_{\text {blea }}$ respectively, on behalf of the sensor nodes $A$, $\mathrm{B}$ detect the arrival and departure time of the vehicle, the average time tand the average time difference $\overline{\Delta t}$ of Vehicles through the A, B two sensor nodes are:

$$
\begin{aligned}
& \overline{\mathrm{t}}=\frac{\left(\mathrm{t}_{\text {alea }}-\mathrm{t}_{\text {aarr }}\right)+\left(\mathrm{t}_{\text {blea }}-\mathrm{t}_{\text {blea }}\right)}{2} \\
& \overline{\Delta \mathrm{t}}=\frac{\left(\mathrm{t}_{\text {barr }}-\mathrm{t}_{\text {aarr }}\right)+\left(\mathrm{t}_{\text {blea }}-\mathrm{t}_{\text {alea }}\right)}{2} .
\end{aligned}
$$

So the relative vehicle length is:

$$
\mathrm{L}=\frac{\overline{\mathrm{t}}}{\overline{\Delta \mathrm{t}} \mathrm{s}} \text {, }
$$

Where $s$ is the distance between sensor nodes A and B.

(2) The normalized square wave peak (trough) number

The wave peak(trough) is normalized to Square Wave $(1,0,-1)$. In this article, the square wave inputs are decided by the slope of the $\mathrm{X}(\mathrm{n})$. The normalized square waveform is shown in Fig.2. The analysis shows that the normalized peak (trough) corresponds to the sampling point that is serious distortion of the geomagnetic signal of $\mathrm{X}$ axis, which is the moment that engine or engine shaft passes through the sensor nodes[7]. Therefore, the peak (trough) number $\mathrm{N}$ can reflect the axle number.
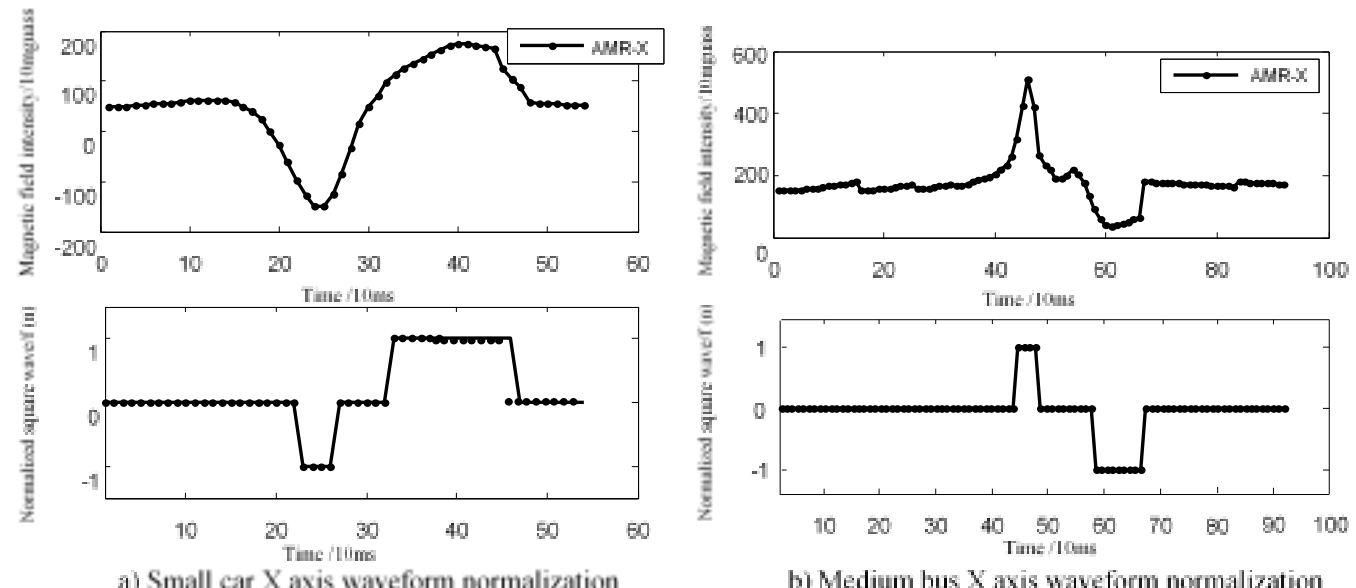

b) Medium bus $\mathrm{X}$ axis waveform normalization

Figure2.Normalized waveforms of different vehicle types

(3) The total energy of vehicle signal

The total energy of the vehicle signal reflects the wave disturbance magnitude produced by the vehicle's passing, so waveform total energy can be used to classify vehicle models. The formula for the calculation of the total energy waveform is:

$$
\mathrm{E}=\sum\left(\mathrm{X}(\mathrm{n})^{2}+\mathrm{Y}(\mathrm{n})^{2}+\mathrm{Z}(\mathrm{n})^{2}\right) .
$$


(4) The average energy of vehicle signal

Similar to total energy, the average energy can also reflect the degree of distortion of the disturbance signal. The average energy calculation formula is:

$$
\mathrm{E}_{\mathrm{v}}=\frac{\mathrm{E}}{\mathrm{L}}
$$

\section{Vehicle Classification Algorithm}

Hierarchical classification tree is constructed by the four features: Relative vehicle length, total energy, average energy, normalized number of the peaks and valleys. It classifies the vehicles into four types. The specific classification process is shown in Fig.3.

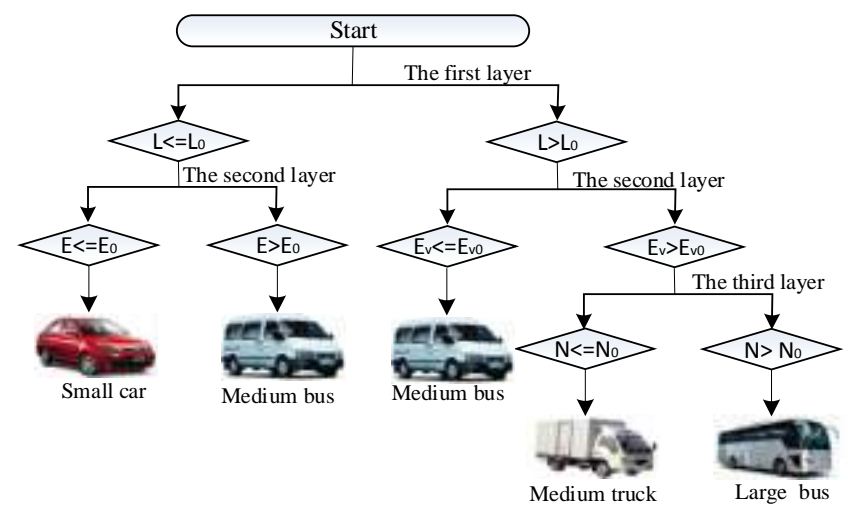

Figure3. Hierarchical decision tree classification process

The decision tree is divided into three layers. The classification standard of first layer is the relative length of the vehicle $\mathrm{L}$. The second is vehicle energy which contains total energy Eand average $\mathrm{E}_{\mathrm{V}}$. The third is the number of the peaks (troughs)N. $\left\{\mathrm{L}_{0}, \mathrm{E}_{0}, \mathrm{E}_{\mathrm{V} 0}, \mathrm{~N}_{0}\right\}$ is a group of fixed threshold algorithm parameters. Actually in this paper, $\mathrm{L}_{0}=5, \mathrm{E}_{0}=10000, \mathrm{E}_{\mathrm{V} 0}=1000, \mathrm{~N}_{0}=3$. Hierarchical decision tree algorithm is effective in classification with less computation and higher accuracy.

\section{Experimental Results}

Finally, the dual sensor information fusion vehicle detection system was installed in the campus road for information collection. The speed of the road is less than $40 \mathrm{~km} / \mathrm{h}$. The schematic diagram of the sensor arrangement is shown in Fig.4.The data processing of the host was placed on the roadside, and the signal processed by the average filter was sent to the data processing host for the $100 \mathrm{~Hz}$ frequency(sensor node sampling by frequency of $1000 \mathrm{~Hz}$ ). The algorithm was tested and realized in the data processing host. 


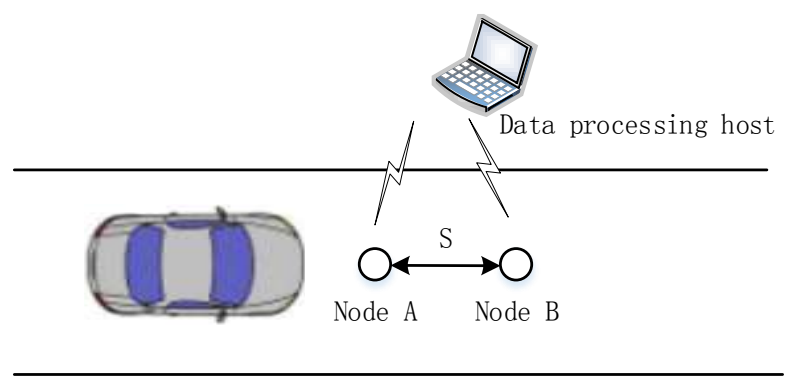

Figure4. Schematic diagram of vehicle detection system with two sensor nodes

In process of experiment, the video camera was used for real-time data shooting, in order to verify the good performance of the algorithm. The model identification results of single sensor node and dual sensor nodes are shown in Table 1.As can be seen from table 1, compared with the single node classification algorithm, the accuracy of the vehicle classification algorithm with the information fusion of the two sensor nodes is higher.

Table 1. The model identification results of single sensor node and dual sensor nodes

\begin{tabular}{cccccccc}
\hline \multirow{2}{*}{$\begin{array}{c}\text { Vehicle type } \\
\text { identification } \\
\text { data }\end{array}$} & $\begin{array}{c}\text { Automatic identification data } \\
\text { A }\end{array}$ & $\begin{array}{c}\text { Node } \\
\text { A }\end{array}$ & $\begin{array}{c}\text { Node } \\
\text { B }\end{array}$ & Dual node & $\begin{array}{c}\text { Node } \\
\text { A }\end{array}$ & Node B & $\begin{array}{c}\text { Dual } \\
\text { node }\end{array}$ \\
\hline $\begin{array}{c}\text { Small car } \\
\text { Medium } \\
\text { bus }\end{array}$ & 198 & 157 & 160 & 165 & $79.2 \%$ & $80.8 \%$ & $83.3 \%$ \\
$\begin{array}{c}\text { Medium } \\
\text { truck }\end{array}$ & 116 & 91 & 94 & 99 & $78.4 \%$ & $81 \%$ & $85.3 \%$ \\
Large Bus & 52 & 40 & 39 & 42 & $76.9 \%$ & $75 \%$ & $82.7 \%$ \\
\hline
\end{tabular}

\section{Conclusions}

A vehicle classification algorithm based on double sensor information fusion is proposed in this paper.The vehicle information is obtained by using double threshold method, which is fused by the maximum likelihood estimation.Hierarchical decision tree is used to classify the vehicle, which takes the vehicle feature information as input.The vehicle detection system is completed by laying out the sensor nodes in the same lane, which is simple in installation and low in cost, and can be used to classify different types of vehicles. The experimental results show that the proposed algorithm can classify the vehicle more accurately through integrating the vehicle information detected from different sensor nodes in the same segment.

\section{References}

[1] Xiao-sen Zhang. Research and Implementation of Vehicle Detection Algorithm Based on Megnetic Resistance Sensors[D]. Beijing Jiaotong University,2012. In Chinese 
[2] Kaewkamnerd S, Chinrungrueng J, Pongthornseri R, et al. Vehicle classification based on magnetic sensor signal[C]. Information and Automation (ICIA), 2010 IEEE International Conference on. IEEE, 2010: 935-939.

[3] Yang B, Lei Y.[J]IEEE Sensors Journal, 2015, 15(2): 1132-1138.

[4] Bo-gen Cai,Jimin Zhao, Wang Jian, et al. [J]Journal of Transportation Systems Engineering and Information Technology,2014,04:46-52. In Chinese

[5] He Y, Du Y, Sun L. [J]Procedia-Social and Behavioral Sciences, 2012, 43: 618-627.

[6] Meta S, Cinsdikici M G. [J] IEEE Transactions on Vehicular Technology, 2010, 59(6): 2795-2805.

[7] Xu Lei, Ying-yingHou, Qi-sheng Wu. [J]Journal of Chang'an University(Natural Science Edition), 2013, 5: 022.In Chinese 